\title{
GENERALIZED FINITE ELEMENT METHOD FOR VIBRATION ANALYSIS OF BARS
}

\author{
M. Arndt ${ }^{1}$, A. J. Torii ${ }^{1,2}$, R. D. Machado ${ }^{1}$, A. Scremin ${ }^{1}$ \\ ${ }^{1}$ Numerical Methods in Engineering Graduated Program, Federal University of Paraná \\ (arndt@ufpr.br) \\ ${ }^{2}$ Universidade Positivo (ajtorii@up.com.br)
}

\begin{abstract}
The vibration analysis is an important stage in the design of mechanical systems and structures subject to dynamic loads like wind and earthquake. The Finite Element Method (FEM) is commonly used in vibration analysis and its approximated solution can be improved using two refinement techniques: $h$ and p-versions. The h-version of FEM gives good results for the lowest frequencies but demands great computational cost to work up the accuracy for the higher frequencies. The accuracy of the FEM can be improved applying the polynomial $p$ refinement. Some enriched methods based on the FEM have been developed in last 20 years seeking to increase the accuracy of the solutions for the higher frequencies with lower computational cost. The purpose of this paper is to present a formulation of the Generalized Finite Element Method (GFEM) to free and transient vibration analysis of bars. The Generalized Finite Element Method is developed by enriching the standard Finite Element Method space, whose basis performs a partition of unity, with knowledge about the differential equation being solved. The proposed method combines the best features of GFEM and enriched methods: (a) efficiency, (b) hierarchical refinements and (c) the introduction of boundary conditions following the standard finite element procedure. In addition the enrichment functions are easily obtained. The main features of the GFEM are discussed and the partition of unity functions and the local approximation spaces are presented. The efficiency and convergence of the proposed method for vibration analysis of bars are checked. The results obtained by the GFEM are compared with those obtained by the analytical solution, some enriched methods and the $h$ and $p$ versions of the Finite Element Method.
\end{abstract}

Keywords: Generalized finite element method, Dynamic analysis, Vibration analysis, Partition of unity.

\section{INTRODUCTION}

The dynamic analysis is an important stage in the design of mechanical systems and structures subject to dynamic loads like wind and earthquake. This analysis allows obtaining 
the dynamic characteristics and the time-varying response of these structures. The dynamic analysis can be used also to identify cracks in structures.

The Finite Element Method (FEM) is commonly used in vibration analysis and its approximated solution can be improved using two refinement techniques: $h$ and $p$-versions. The $h$-version consists of the refinement of element mesh; the $p$-version may be understood as the increase in the number of shape functions in the element domain without any change in the mesh. The conventional $p$-version of FEM consists of increasing the polynomial degree in the solution. The $h$-version of FEM gives good results for the lowest frequencies but demands great computational cost to work up the accuracy for the higher frequencies. The accuracy of the FEM can be improved applying the polynomial $p$ refinement.

Some enriched methods based on the FEM have been developed in last 20 years seeking to increase the accuracy of the solutions for the higher frequencies with lower computational cost. Engels [8] and Ganesan \& Engels [9] present the Assumed Mode Method (AMM) which is obtained adding to the FEM shape functions set, some interface restrained assumed modes. The Composite Element Method (CEM) [23, 24] is obtained by enrichment of the conventional FEM local solution space with non-polynomial functions obtained from analytical solutions of simple vibration problems. A modified CEM applied to analysis of beams is proposed by [12]. The use of products between polynomials and Fourier series instead of polynomials alone in the element shape functions is recommended by [11]. They develop the Fourier $p$-element applied to the vibration analysis of bars, beams and plates. These three methods have the same characteristics and they will be called enriched methods in this work. The main features of the enriched methods are: (a) the introduction of boundary conditions follows the standard finite element procedure; (b) hierarchical $p$ refinements are easily implemented and (c) they are more accurate than conventional $h$ version of FEM.

At the same time, the Generalized Finite Element Method (GFEM) was independently proposed by Babuska and colleagues [2, 6, 13] and by Duarte \& Oden [7, 14] under the following names: Special Finite Element Method, Generalized Finite Element Method, Finite Element Partition of Unity Method, $h p$ Clouds and Cloud-Based $h p$ Finite Element Method. Actually, several meshless methods recently proposed may be considered special cases of this method. Strouboulis and co-workers [19] define otherwise the subclass of methods developed from the Partition of Unity Method including $h p$ Cloud Method [7, 14], the eXtended Finite Element Method (XFEM) [20, 21], the Generalized Finite Element Method (GFEM) [17, 18], the Method of Finite Spheres [4], and the Particle-Partition of Unity Method [16]. The GFEM, which was conceived on the basis of the Partition of Unity Method, allows the inclusion of a priori knowledge about the fundamental solution of the governing differential equation. This approach ensures accurate local and global approximations. In structural dynamics, the Partition of Unity Method was applied by [5] and [10] to numerical vibration analysis of plates and by [1] to free vibration analysis of bars and trusses. Among the main challenges in developing the GFEM to a specific problem are: (a) choosing the appropriate space of functions to be used as local approximation and (b) the imposition of essential boundary conditions, since the degrees of freedom used in GFEM generally do not correspond to the nodal ones. In most cases the imposition of boundary conditions is achieved by the 
degeneration of the approximation space or applying penalty methods or Lagrange multipliers.

The purpose of this work is to present a formulation of the GFEM to free and transient vibration analysis of bars. The proposed method combines the best features of GFEM and enriched methods: (a) efficiency, (b) hierarchical refinements and (c) the introduction of boundary conditions following the standard finite element procedure. In addition the enrichment functions are easily obtained.

\section{GENERALIZED FINITE ELEMENT METHOD}

The Generalized Finite Element Method (GFEM) is a Galerkin method whose main goal is the construction of a finite dimensional subspace of approximating functions using local knowledge about the solution that ensures accurate local and global results. The GFEM local enrichment in the approximation subspace is incorporated by the partition of unity approach.

\subsection{Partition of unity}

Let $u \in \mathrm{H}^{1}(\Omega)$ be the function to be approximated and $\left\{\Omega_{i}\right\}$ be an open cover of domain $\Omega$ (Fig. 1) satisfying an overlap condition:

$$
\exists M_{S} \in \mathbf{N} \text { so that } \forall x \in \Omega \quad \operatorname{card}\left\{i \mid x \in \Omega_{i}\right\} \leq M_{S} .
$$

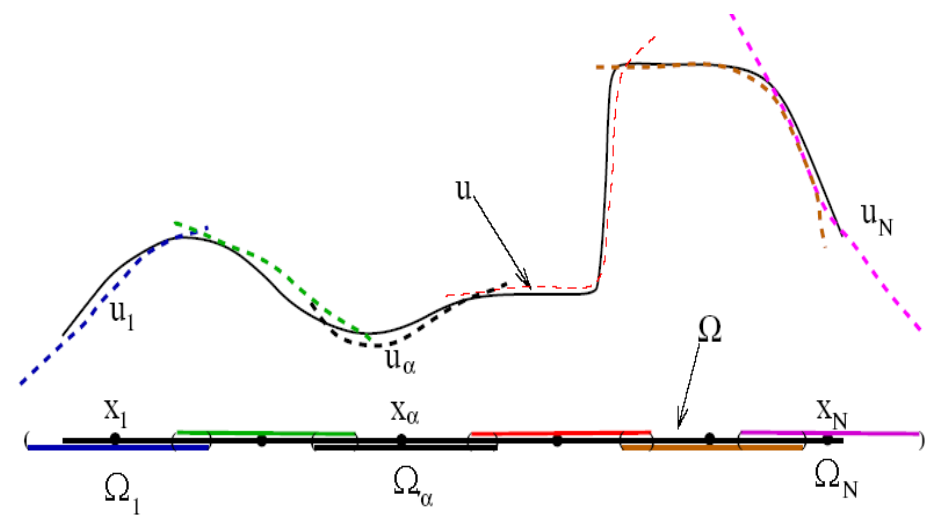

Figure 1. Open cover $\left\{\Omega_{i}\right\}$ of domain $\Omega$ (see [6]).

A Lipschitz partition of unity subordinate to the cover $\left\{\Omega_{i}\right\}$ is the set of functions $\left\{\eta_{i}\right\}$ satisfying the conditions:

$$
\begin{gathered}
\operatorname{supp}\left(\eta_{i}\right)=\left\{x \in \Omega \mid \eta_{i}(x) \neq 0\right\} \subset\left[\Omega_{i}\right], \quad \forall i, \\
\sum_{i} \eta_{i} \equiv 1 \text { on } \Omega,
\end{gathered}
$$


where $\operatorname{supp}\left(\eta_{i}\right)$ denotes the support of definition of the function $\eta_{i}$ and $\left[\Omega_{i}\right]$ is the closure of the patch $\Omega_{i}$.

The partition of unity set $\left\{\eta_{i}\right\}$ allows obtaining an enriched set of approximating functions. Let $S_{i} \subset \mathrm{H}^{1}\left(\Omega_{i} \cap \Omega\right)$ be a set of functions that locally well represents $u$ :

$$
S_{i}=\left\{S_{i}^{j}\right\}_{j=1}^{m}
$$

Then the enriched set is formed by multiplying each partition of unity function $\eta_{i}$ by the corresponding $s_{i}^{j}$, i.e.,

$$
S:=\sum_{i} \eta_{i} S_{i}=\left\{\sum_{i} \eta_{i} s_{i}^{j} \mid s_{i}^{j} \in S_{i}\right\} \subset H^{1}(\Omega) .
$$

Accordingly, the function $u$ can be approximated by the enriched set as:

$$
u_{h}(x)=\sum_{i} \sum_{s_{i}^{j} \in S_{i}} \eta_{i} s_{i}^{j}(x) a_{i j} .
$$

where $a_{i j}$ are the degrees of freedom.

In the proposed GFEM, the cover $\left\{\Omega_{i}\right\}$ corresponds to the finite element mesh and each patch $\Omega_{i}$ corresponds to the sub domain of $\Omega$ formed by the union of elements that contain the node $x_{i}$ (Fig. 2).

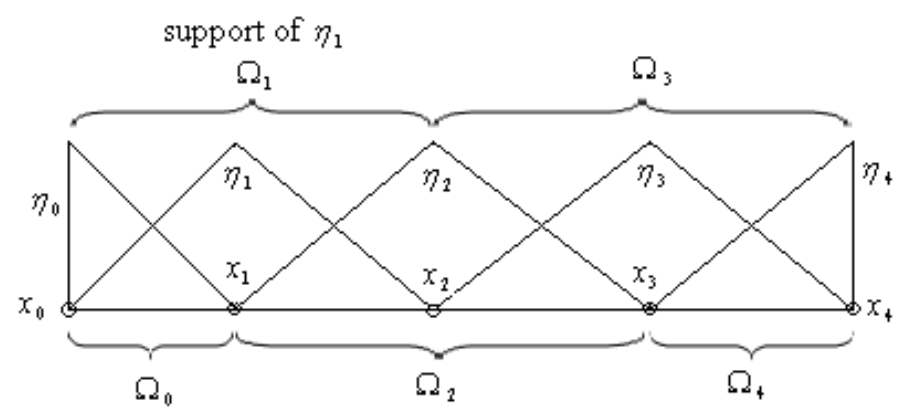

Figure 2. Patchs and partition of unity set for one-dimensional GFEM finite element mesh

\subsection{Generalized $C^{0}$ elements for free vibration analysis}

The generalized $\mathrm{C}^{0}$ elements use the classical linear FEM shape functions as the partition of unity, i.e.:

$$
\eta_{i}= \begin{cases}1+\frac{x-x_{i}}{x_{i}-x_{i-1}} & \text { if } x \in\left(x_{i-1}, x_{i}\right) \\ 1-\frac{x-x_{i}}{x_{i+1}-x_{i}} & \text { if } x \in\left(x_{i}, x_{i+1}\right)\end{cases}
$$


in the patch $\Omega_{i}=\left(x_{i-1}, x_{i+1}\right)$.

The proposed local approximation space in the patch $\Omega_{i}=\left(x_{i-1}, x_{i+1}\right)$ takes the form:

$$
\begin{aligned}
& S_{i}=\operatorname{span}\left\{\begin{array}{cccccc}
1 & \gamma_{1 j} & \gamma_{2 j} & \varphi_{1 j} & \varphi_{2 j} & \ldots
\end{array}\right\}, j=1,2, \ldots, n_{l}, \\
& \gamma_{1 j}=\left\{\begin{array}{c}
0 \text { if } x \in\left(x_{i-1}, x_{i}\right) \\
\sin \left[\beta_{R j}\left(x-x_{i}\right)\right] \text { if } x \in\left(x_{i}, x_{i+1}\right),
\end{array}\right. \\
& \gamma_{2 j}=\left\{\begin{array}{c}
\sin \left[\beta_{L j}\left(x-x_{i}\right)\right] \text { if } \quad x \in\left(x_{i-1}, x_{i}\right) \\
0 \text { if } x \in\left(x_{i}, x_{i+1}\right)
\end{array},\right. \\
& \varphi_{1 j}=\left\{\begin{array}{c}
0 \text { if } x \in\left(x_{i-1}, x_{i}\right) \\
\cos \left[\beta_{R j}\left(x-x_{i}\right)\right]-1 \text { if } x \in\left(x_{i}, x_{i+1}\right)
\end{array}\right. \\
& \varphi_{2 j}=\left\{\begin{array}{c}
\cos \left[\beta_{L j}\left(x-x_{i}\right)\right]-1 \quad \text { if } \quad x \in\left(x_{i-1}, x_{i}\right) \\
0 \text { if } x \in\left(x_{i}, x_{i+1}\right)
\end{array},\right. \\
& \beta_{R j}=\sqrt{\frac{\rho_{R}}{E_{R}}} \mu_{j} \\
& \beta_{L j}=\sqrt{\frac{\rho_{L}}{E_{L}}} \mu_{j}
\end{aligned}
$$

where $E_{R}$ and $\rho_{R}$ are the Young modulus and specific mass on sub domain $\left(x_{i}, x_{i+1}\right), E_{L}$ and $\rho_{L}$ are the Young modulus and specific mass on sub domain $\left(x_{i-1}, x_{i}\right)$, and $\mu_{j}$ is a frequency related to the enrichment level $j$.

The enriched set $S$, so proposed, vanishes at element nodes, which allows the imposition of boundary conditions in the same fashion of the finite element procedure.

This $\mathrm{C}^{0}$ element can be applied in the free vibration analysis of shafts, bars and trusses. Different frequencies $\mu_{j}$ produce different enriched elements. The increase in the number of elements in the mesh with only one level of enrichment $(j=1)$ and a fixed parameter $\beta_{1}=\beta_{R 1}=\beta_{L 1}$, for example $\beta_{1}=\pi$, produces an $h$ refinement. Otherwise the increase in the number of levels of enrichment, with a different parameter $\beta_{j}=\beta_{R j}=\beta_{L j}$ each, for example, $\beta_{j}=j \pi$, produces a hierarchical $p$ refinement. Another refinement possible in the proposed GFEM is the adaptive refinement, which is presented below.

The adaptive GFEM is an iterative approach presented first by [1] whose main goal is to increase the accuracy of the frequency (eigenvalue) related to a chosen vibration mode with order denoted by "target order". The flowchart with blocks A to H presented in Figure 3 represents the adaptive process. 


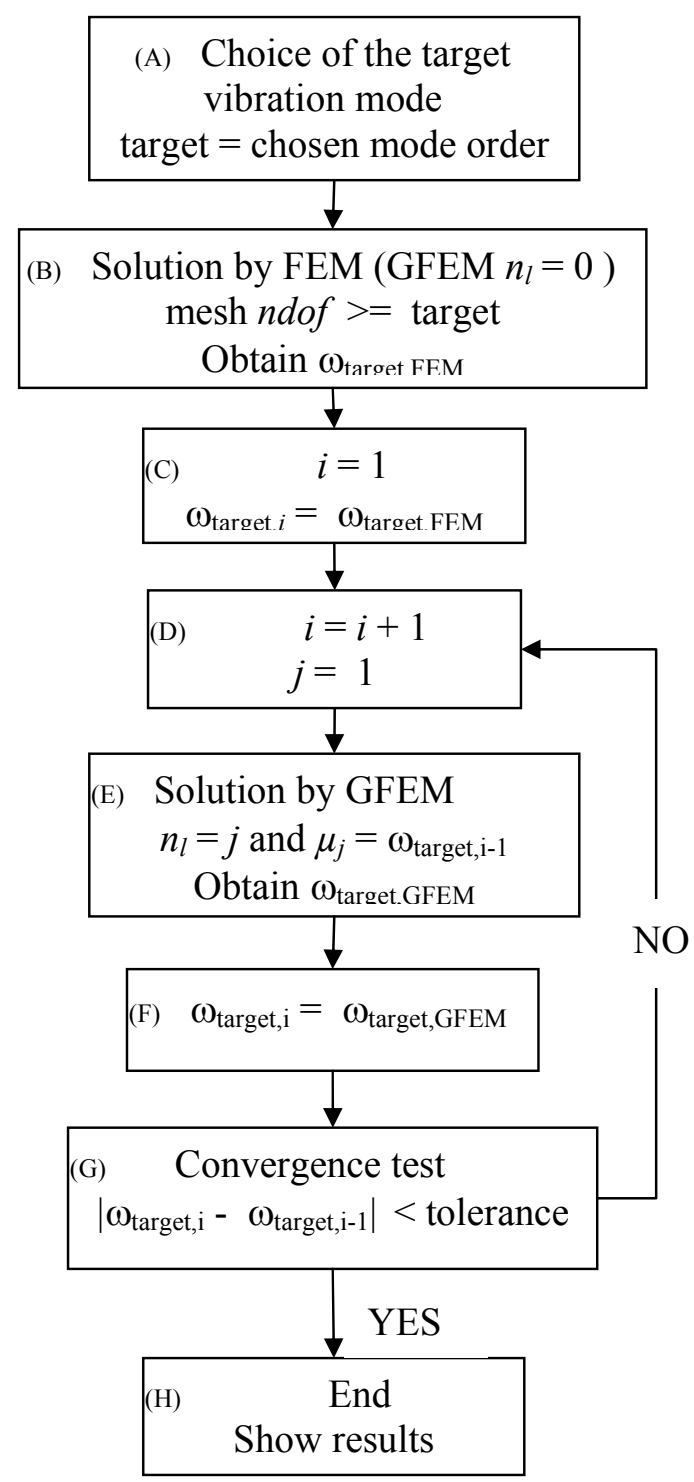

Figure 3. Flowchart of the adaptive GFEM.

In this flowchart, $\omega_{\text {target }}$ corresponds to the frequency related to the target mode. The first step of the adaptive GFEM process (blocks A to C) consists in obtaining an approximation of the target frequency by the standard FEM (GFEM with $n_{l}=0$ ) with a coarse mesh. The finite element mesh used in the analysis has to be as coarse as is necessary to capture a first approximation of the target frequency. The subsequent steps (blocks D to G) consist in applying the GFEM with only one enrichment level $\left(n_{l}=1\right)$ to the same finite element mesh assuming the frequency $\mu_{j}(j=1$, blocks $\mathrm{D}$ and $\mathrm{E})$ of the enrichment functions (Eqs. 9-14) as the target frequency obtained in the last step. Thus, no mesh refinement is necessary along the iterative process.

Both the standard FEM and the adaptive GFEM allow as many frequencies as the total number of degrees of freedom to be obtained. However, in the latter, only the precision of the target frequency is effectively improved by the iterative process. The other frequencies 
present errors similar to those obtained by the standard FEM with the same mesh. In order to improve the precision of another frequency, it is necessary to perform a new adaptive GFEM analysis, taking this new one as the target frequency.

\subsection{Generalized $C^{0}$ elements for time response analysis}

The $\mathrm{C}^{0}$ elements described in the previous section can also be used for time response analysis. When damping is not considered, time response analysis can be made by solving the following system of equations [3]:

$$
\mathbf{K u}+\mathbf{M u ̈}=\mathbf{F},
$$

where $\mathbf{u}$ is the vector of displacements, $\ddot{\mathbf{u}}$ is the vector of accelerations, $\mathbf{K}$ is the stiffness matrix, $\mathbf{M}$ is the mass matrix and $\mathbf{F}$ is the vector of applied forces.

The system of equations from Eq. (15) can be solved by some time integration scheme found in the literature. In this paper, Modal Superposition is used to obtain a set of independent equations from Eq. (15) and then each equation is solved separately by Newmark's Method [3]. The advantage of using this approach is that one is able to choose which vibration modes to include in the Modal Superposition procedure. This can be an interesting tool to investigate the accuracy of the individual approximated vibration modes of the structure.

The error between a reference solution and an approximate solution in a fixed position inside the structure can be approximated by

$$
e \approx \sum_{i=1}^{n_{t}} \Delta t\left|u^{(i)}-u_{h}^{(i)}\right|
$$

where $n_{t}$ is the number of time steps used, $\Delta t$ is the time step used to obtain the approximate solution, $u^{(i)}$ is the analytical solution at time step $(i)$ and $u_{h}{ }^{(i)}$ is the approximate solution at time step $(i)$ in a fixed position inside the structure. Error evaluation according to Eq. (16) is illustrated in Fig. 4. The error in a given time interval is approximated by the product between $\Delta t$ and $\Delta u^{(i)}$.

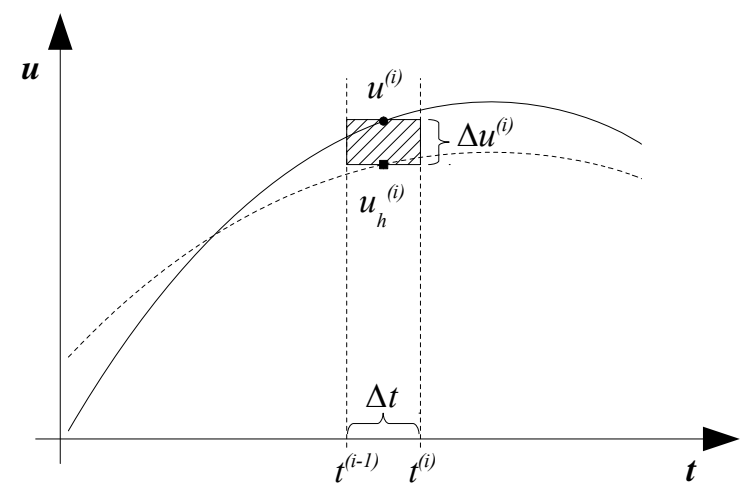

Figure 4: Error evaluation according to Eq. (16). 
For the time response analysis, higher order polynomial finite elements were obtained using Lobatto's polynomials as shape functions, as described in details by [22]. These polynomials are different from Lagrange's polynomials that are commonly used in $p$-version of FEM. However, both families of polynomials form a basis for the subspace $P_{n}$ of polynomials up to order $n$ when $n+1$ shape functions are used. Consequently, the approximations given by both schemes are the same.

\section{APPLICATION}

Numerical solutions for a uniform fixed-free bar (Fig. 5) are given below to check the efficiency of the proposed formulation of GFEM.

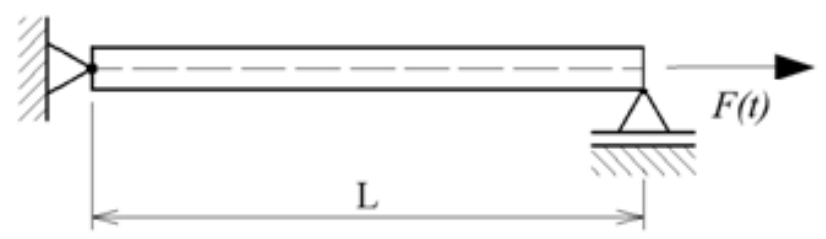

Figure 5. Uniform fixed-free bar.

The number of degrees of freedom (ndof) considered in each analysis is the total number of effective degrees of freedom after introduction of boundary conditions.

\subsection{Free vibration analysis}

The free axial vibration of a fixed-free bar (Fig. 5 with $F(t)=0$ ) with length $L$, elasticity modulus $E$, mass density $\rho$ and uniform cross section area $A$, has exact natural frequencies $\left(\omega_{r}\right)$ given by:

$$
\omega_{r}=\frac{(2 r-1) \pi}{2 L} \sqrt{\frac{E}{\rho}}, \quad r=1,2, \ldots
$$

In order to compare the exact solution with the approximated ones, in this example a non-dimensional eigenvalue $\chi_{r}$ given by:

$$
\chi_{r}=\frac{\rho L^{2} \omega_{r}^{2}}{E}
$$

will be used.

To check the efficiency of the $p$ refinement of GFEM the results were compared to those obtained by AMM, by CEM, by Fourier, by linear and cubic $h$-versions of FEM and by conventional $p$-version of FEM. The shape functions of the conventional $p$-version of FEM are Lagrangian polynomials. The $p$-version of GFEM consists in a progressive increase of levels of enrichment with parameter $\beta_{j}=j \pi$. In the analyses by $p$-version of FEM and by all 
the enriched methods, the bar was described geometrically by one element and the successive refinements were obtained increasing the number of shape functions. Figures 6 to 8 present the behavior of relative error for the six earliest eigenvalues in logarithmic scale.

Analyzing the results obtained for the fixed-free bar, one observes that the results obtained by GFEM and all enriched methods show convergence rates greater than the linear $h$ refinement of the FEM. The cubic $h$-version of FEM shows better results than CEM / Fourier and AMM just for three earliest eigenvalues and it shows worst results than GFEM for all eigenvalues. The conventional hierarchical $p$ refinement of the FEM has greater accuracy than CEM / Fourier and AMM. Otherwise, the GFEM showed worst precision than the $p$ version of the FEM only for the first eigenvalue.
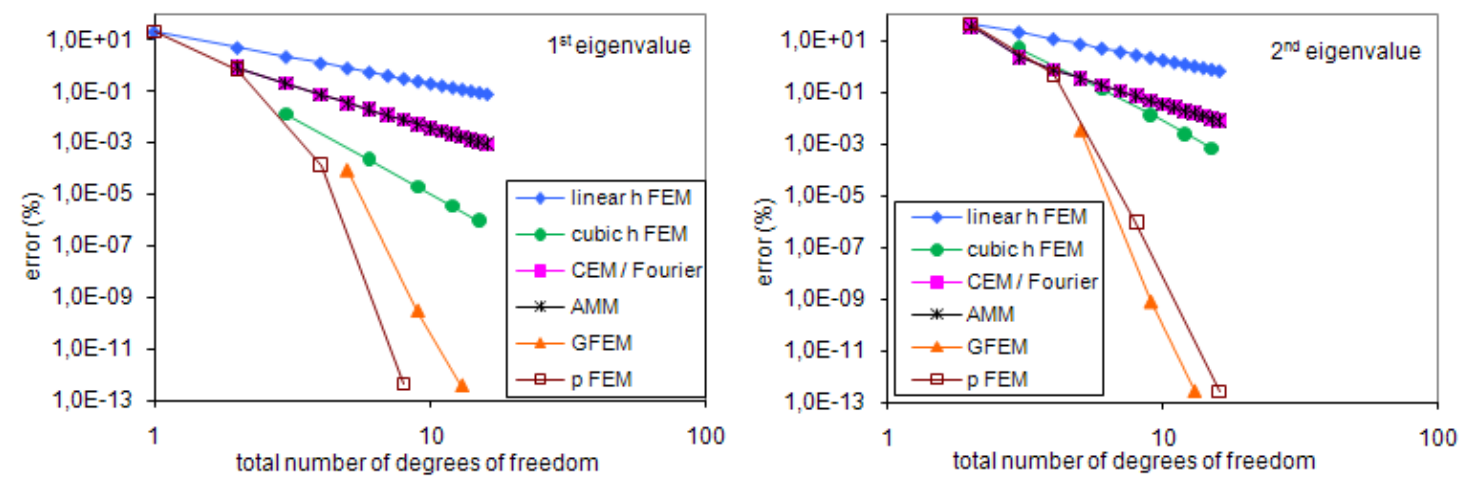

Figure 6. Relative error (\%) for the $1^{\text {st }}$ and $2^{\text {nd }}$ bar eigenvalues.
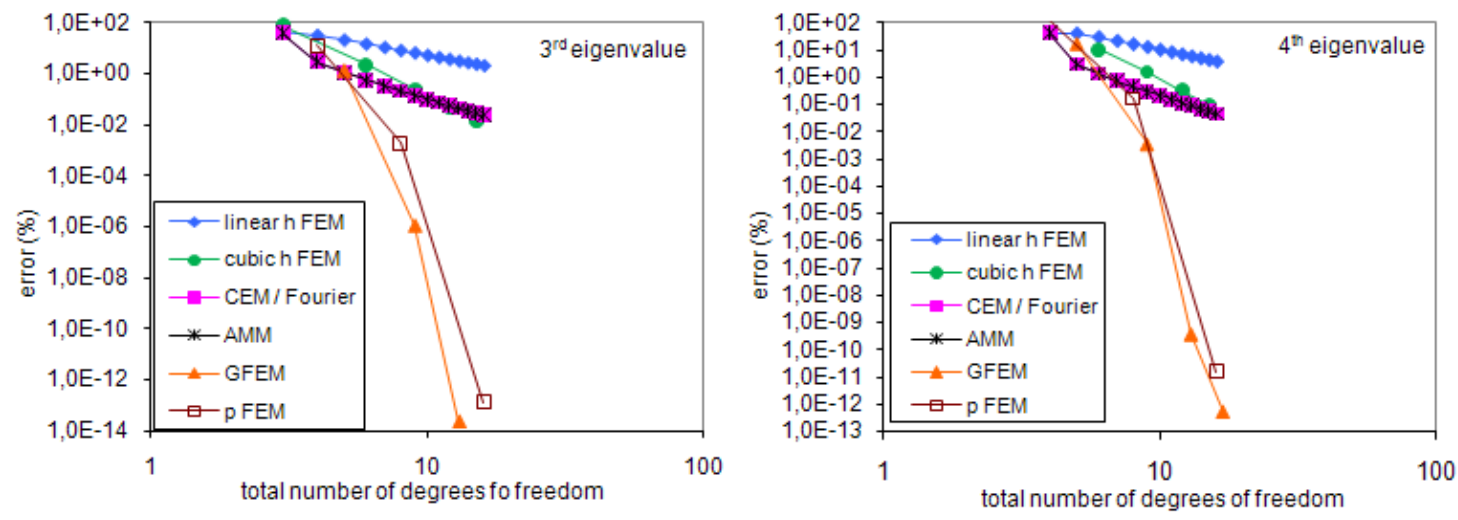

Figure 7. Relative error $(\%)$ for the $3^{\text {rd }}$ and $4^{\text {th }}$ bar eigenvalues. 

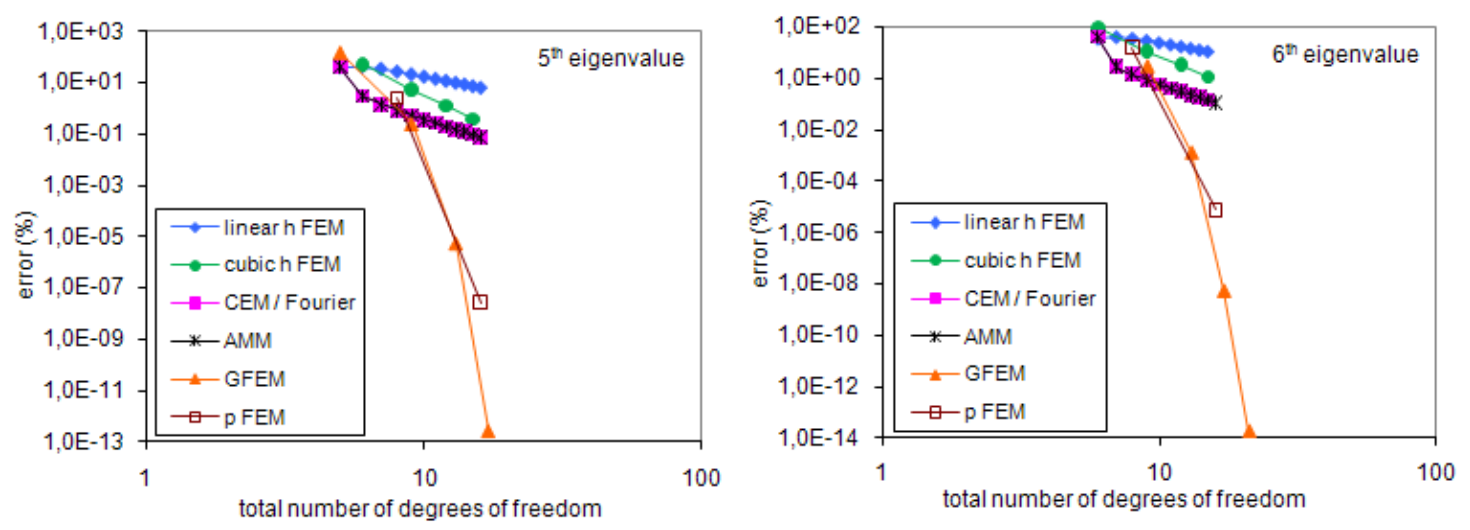

Figure 8. Relative error $(\%)$ for the $5^{\text {th }}$ and $6^{\text {th }}$ bar eigenvalues.

Four different adaptive GFEM analyses are performed in order to obtain the first four frequencies. In order to capture an initial approximation of the target vibration frequency, for the first frequency, the finite element mesh must have at least one bar element (one effective degree of freedom), for the second frequency, it must have at least two bar elements (two effective degrees of freedom), and so on.

Table 1 presents the relative errors obtained by the numerical methods. The linear FEM solution is obtained with 100 elements, that is, 100 effective degrees of freedom (dof). The cubic FEM solution is obtained with 20 elements, that is, 60 effective degrees of freedom. The CEM solution is obtained with one element and 15 enrichment functions corresponding to one nodal degree of freedom and 15 field degrees of freedom resulting in 16 effective degrees of freedom. The conventional hierarchical $p$ FEM solution is obtained with a 17-node element corresponding to 16 effective degrees of freedom. The analyses by the adaptive GFEM have no more than 20 degrees of freedom in each iteration. For example, the fourth frequency is obtained taking 4 degrees of freedom in the first iteration and 20 degrees of freedom in the two subsequent ones.

Table 1. Results to free vibration of uniform fixed-free bar

\begin{tabular}{|c|c|c|c|c|c|c|}
\hline \multirow[t]{2}{*}{ Eigenvalue } & $\begin{array}{c}\text { linear } h \\
\text { FEM } \\
(100 \mathrm{e}) \\
\text { ndof }=100\end{array}$ & $\begin{array}{c}\text { cubic } h \\
\text { FEM } \\
(20 \mathrm{e}) \\
\text { ndof }=60\end{array}$ & $\begin{array}{c}p \text { FEM } \\
(1 \mathrm{e} 17 \mathrm{n}) \\
\text { ndof }=16\end{array}$ & $\begin{array}{c}\text { CEM } \\
(1 \mathrm{e} 15 \mathrm{c}) \\
\text { ndof }=16\end{array}$ & \multicolumn{2}{|c|}{$\begin{array}{l}\text { Adaptive GFEM } \\
\text { (after } 3 \text { iterations) }\end{array}$} \\
\hline & error (\%) & error $(\%)$ & error $(\%)$ & error $(\%)$ & error $(\%)$ & ndof in iterations \\
\hline 1 & $2,056 \mathrm{e}-3$ & 8,564 e- 10 & 3,780 e- 13 & 8,936 e-4 & 3,780 e -13 & $1 \times 1$ dof $+2 \times 5$ dof \\
\hline 2 & $1,851 \mathrm{e}-2$ & 1,694 e-7 & 2,560 e- 13 & 8,188 e-3 & 2,560 e- 13 & $1 \times 2$ dof $+2 \times 10$ dof \\
\hline 3 & $5,141 \mathrm{e}-2$ & 3,619 e- 6 & 1,382 e- 13 & 2,299 e-2 & 2,304 e-14 & $1 \times 3$ dof $+2 \times 15$ dof \\
\hline 4 & 1,008 e- 1 & $2,711 \mathrm{e}-5$ & 1,602 e- 11 & $4,579 \mathrm{e}-2$ & 5,289 e- 13 & $1 \times 4$ dof $+2 \times 20$ dof \\
\hline
\end{tabular}

The adaptive process converges rapidly, requiring three iterations in order to achieve each target frequency with precision of the $10^{-13}$ order. For the uniform fixed-free bar, one notes that the adaptive GFEM reaches greater precision than the $h$ versions of FEM and the CEM. The $p$-version of FEM is as precise as the adaptive GFEM only for the first two eigenvalues. After this, the precision of the adaptive GFEM prevails among the others. 


\subsection{Time response analysis}

Time response analysis is made for the structure from Figure 5. This is a fixed-free uniform bar subject to a time dependent force $F(t)$ at the right end. Only axial displacements are considered here.

The properties of the material for this example were chosen to give a wave velocity equal to $c=\sqrt{E / \rho}=1 \mathrm{~m} / \mathrm{s}$, in order to simplify the analysis. Besides, the length of the bar is taken equal to 1 meter. The bar is at rest and then both initial displacements and velocities are null.

The natural vibration frequencies for this example can be found using Eq. (17). For the time response we assume that the time dependent force is given by

$$
F(t)=f \sin (\omega t),
$$

where $f$ is the force magnitude and $\omega$ is the excitation frequency.

The analytical solution for the time response in this case requires the application of techniques described by [15]. The displacements inside the bar are given by

$$
u(x, t)=f x \sin (\omega t)+f \sum_{n=1}^{\infty}\left\{\sin \left(k_{n} x\right)\left[C_{n} \sin \left(k_{n} c t\right)+B_{n}(t)\right]\right\},
$$

where

$$
\begin{gathered}
C_{n}=-\frac{A_{n} \omega}{k_{n} c}, \\
B_{n}(t)=\frac{A_{n} \omega^{2} \sin (\omega t)}{c^{2} k_{n}^{2}-\omega^{2}}-\frac{A_{n} \omega^{3} \sin \left(k_{n} c t\right)}{c^{3} k_{n}^{3}-c k_{n} \omega^{2}}, \\
A_{n}=-\frac{2\left[k_{n} \cos \left(k_{n}\right)-\sin \left(k_{n}\right)\right]}{k_{n}^{2}}, \\
k_{n}=\pi\left(n-\frac{1}{2}\right) .
\end{gathered}
$$

This problem is solved numerically for $\omega=20 \mathrm{rad} / \mathrm{s}$ and $f=1 \mathrm{~N} / \mathrm{m}^{2}$. The analysis is made using the Modal Superposition Method for a time interval of $20 \mathrm{~s}$ and the resulting equations are solved using the Newmark method (with $\alpha=0,5$ and $\delta=0,25$ ) for a time step equal to $1,25 \times 10^{-3} \mathrm{~s}$.

This example was solved by the GFEM, the standard linear FEM and the hierarchical polynomial FEM (Lobatto's shape functions). The mesh used by the linear FEM was obtained by dividing the domain into 20 finite elements of equal size. The mesh used by $p$ FEM was obtained using four finite elements of fifth order. The mesh used by the GFEM was obtained with four finite elements with one enrichment level obtained with $\beta_{R}=\beta_{L}=3 \pi / 2$. All meshes result in 20 degrees of freedom after boundary conditions imposition.

The errors evaluated according to Eq. (16) at the center of the bar (i.e. $x=0,5 \mathrm{~m}$ ) are presented in Table 2, Fig. 9 and Fig. 10. The errors presented in the first column of Table 2 were obtained including only the first vibration mode of the structure in the Modal 
Superposition procedure. The errors presented in the second column were obtained including the first and second modes, and so on.

Table 2. Errors in (m.s) obtained for the time response analysis at $x=0,5 \mathrm{~m}$ for different numbers of modes included in the Modal Superposition procedure.

\begin{tabular}{l|c|c|c|c|c|c|c|c|c|c}
\hline Modes & $\mathbf{1}$ & $\mathbf{2}$ & $\mathbf{3}$ & $\mathbf{4}$ & $\mathbf{5}$ & $\mathbf{6}$ & $\mathbf{7}$ & $\mathbf{8}$ & $\mathbf{9}$ & $\mathbf{1 0}$ \\
\hline FEM & 1,1813 & 1,1823 & 1,2071 & 1,1772 & 1,1407 & 1,2813 & 1,1676 & 1,1948 & 1,2150 & 1,2019 \\
$p$ FEM & 1,1813 & 1,1820 & 1,2042 & 1,1661 & 1,1259 & 1,1876 & 0,1651 & 0,0778 & 0,0891 & 0,0772 \\
GFEM & 1,1813 & 1,1820 & 1,2042 & 1,1661 & 1,1259 & 1,1876 & 0,1592 & 0,0530 & 0,0498 & 0,0258 \\
\hline
\end{tabular}

\begin{tabular}{l|c|c|c|c|c|c|c|c|c}
\hline Modes & $\mathbf{1 1}$ & $\mathbf{1 2}$ & $\mathbf{1 3}$ & $\mathbf{1 4}$ & $\mathbf{1 5}$ & $\mathbf{1 6}$ & $\mathbf{1 7}$ & $\mathbf{1 8}$ & $\mathbf{1 9}$ \\
\hline FEM & 1,1931 & 1,2000 & 1,2068 & 1,2010 & 1,1964 & 1,2006 & 1,2048 & 1,2005 & 1,1968 \\
$p$ FEM & 0,0817 & 0,0801 & 0,0817 & 0,0802 & 0,0801 & 0,0801 & 0,0889 & 0,0812 & 0,0843 \\
GFEM & 0,0379 & 0,0320 & 0,0346 & 0,0328 & 0,0339 & 0,0335 & 0,0446 & 0,0351 & 0,0435 \\
\hline
\end{tabular}

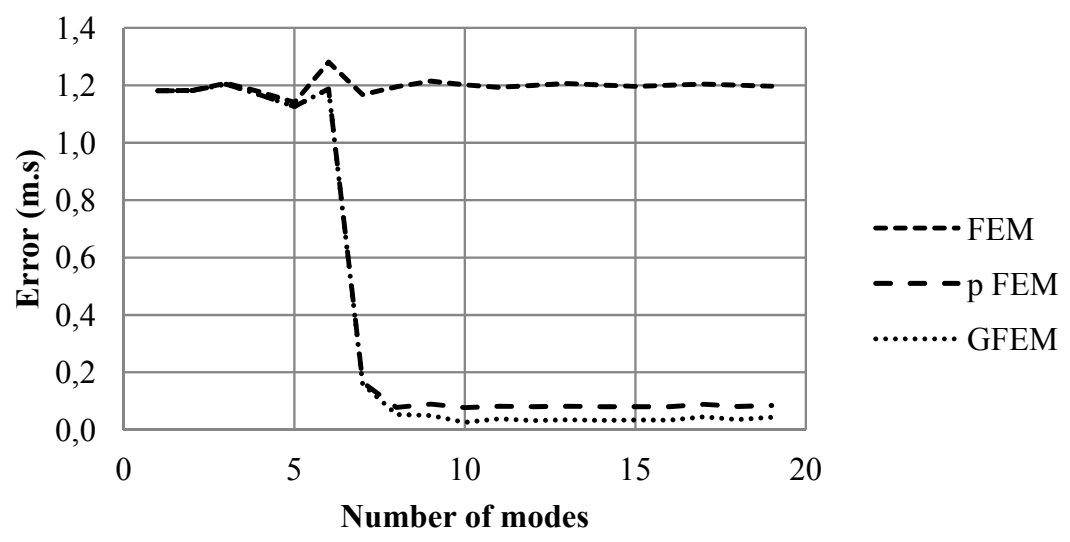

Figure 9. Errors obtained for the time response analysis at $x=0,5 \mathrm{~m}$ for different numbers of modes included in the Modal Superposition procedure.

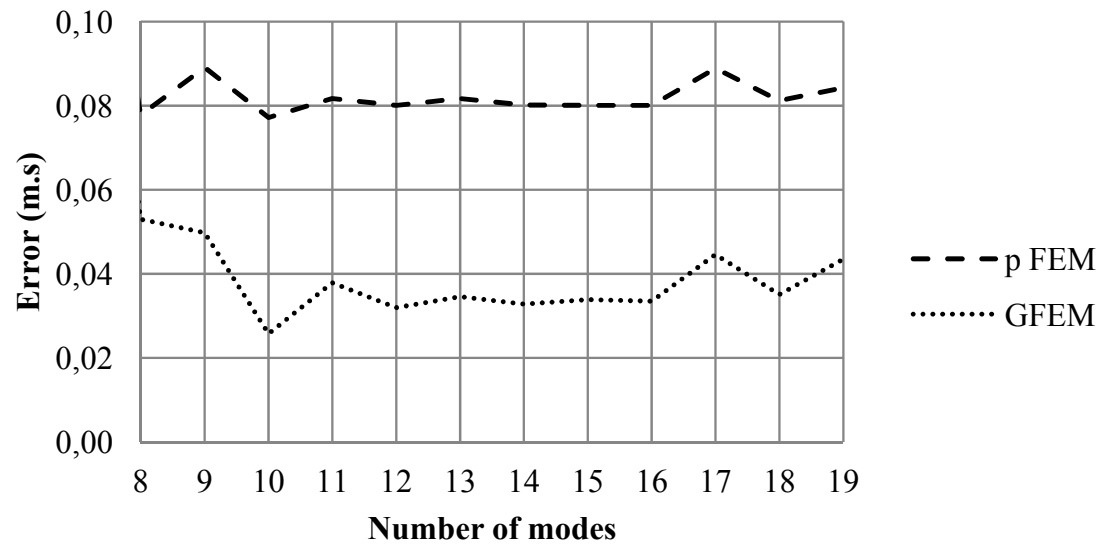

Figure 10. Errors obtained for the time response analysis at $x=0,5 \mathrm{~m}$ for different numbers of modes included in the Modal Superposition procedure. 
The most accurate results were obtained with the GFEM when the first 10 modes were included in the analysis. Besides, the results obtained with the linear FEM are very poor in comparison with the GFEM and the $p$ FEM.

A closer inspection of Figure 10 reveals that including more than 10 modes in the analysis resulted in less accurate results for both the GFEM and the $p$ FEM. This indicates that most accurate results are not obtained by including every mode in Modal Superposition, possibly because the higher modes are poorly approximated. Besides, we note that the errors obtained with the GFEM are significantly smaller then the errors obtained by the $p$ FEM in this case.

This fact is confirmed by observing the time response at $x=0,5 \mathrm{~m}$ obtained by including the first 10 modes in the Modal Superposition procedure, that is presented in Figure 11. We note that the results obtained with the linear FEM are very poor in comparison to the other two methods. The results obtained with the GFEM and the $p$ FEM agree with analytical solution. However, the displacements obtained with the GFEM are much closer to the analytical solution than the ones obtained with the $p$ FEM, mainly for peak displacements.

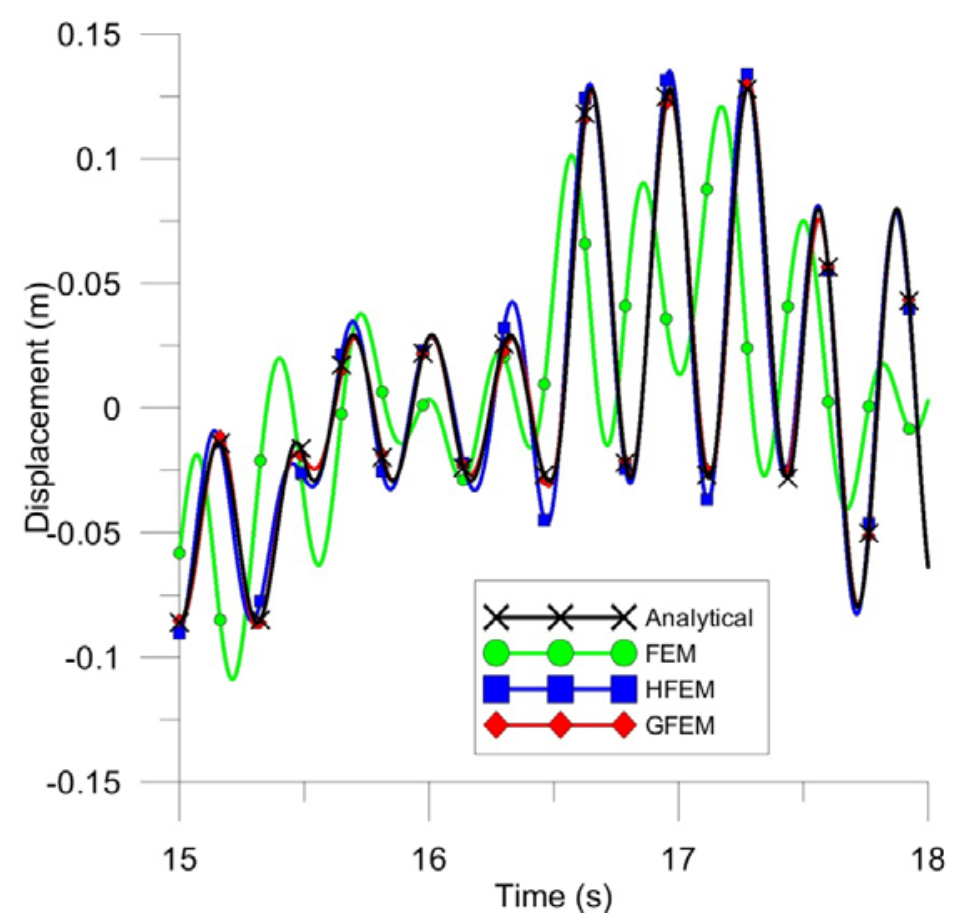

Figure 11 . Time response at $x=0,5 \mathrm{~m}$ obtained by including the first 10 modes in the Modal Superposition procedure.

The accuracy of the results obtained with the GFEM in this case can be explained by observing the relative errors obtained for the natural vibration frequencies. These errors are presented in Table 3, Fig. 12 and Fig. 13.

From Figure 12 we observe that the accuracy of the linear FEM for the approximation of the lower vibration modes is very poor in comparison to the GFEM and the $p$ FEM. The 
linear FEM obtained better results only for the last three frequencies presented, but the errors involved in the approximation of these frequencies are so big that this makes little difference in practice.

The vibration frequencies approximated with errors smaller than $0.05 \%$ are presented in Fig. 13. The results obtained with the linear FEM are not presented since they are very poor in comparison with the other two methods.

In this case, we note an interesting behavior. The $p$ FEM was able to obtain better results for the first few vibration modes, but the results obtained by the GFEM eventually become more accurate for the higher vibration modes. This fact is confirmed by the results presented in Table 3.

However, the approximations obtained by the GFEM are more accurate for a larger range of frequencies. Note, for example, that the GFEM obtained more vibration frequencies with errors smaller than $0.01 \%$. The $p$ FEM was able to obtain better results only for the first four vibration frequencies, but the errors obtained by the GFEM for these frequencies are small enough for most practical applications.

Table 3. Relative errors in (\%) obtained for the natural vibration frequencies.

\begin{tabular}{cccccc}
\hline Modes & Frequency $(\mathbf{r a d} / \mathbf{s})$ & $\boldsymbol{p}$ FEM & GFEM & FEM \\
\hline 1 & 1,570796 & $2,34 \mathrm{E}-04$ & $2,34 \mathrm{E}-04$ & $2,57 \mathrm{E}-02$ \\
2 & 4,712389 & $2,34 \mathrm{E}-04$ & $2,36 \mathrm{E}-03$ & $2,32 \mathrm{E}-01$ \\
3 & 7,853982 & $2,34 \mathrm{E}-04$ & $2,78 \mathrm{E}-03$ & $6,43 \mathrm{E}-01$ \\
4 & 10,995574 & $2,34 \mathrm{E}-04$ & $2,05 \mathrm{E}-03$ & $1,26 \mathrm{E}+00$ \\
5 & 14,137167 & $9,41 \mathrm{E}-04$ & $9,41 \mathrm{E}-04$ & $2,09 \mathrm{E}+00$ \\
6 & 17,278760 & $7,18 \mathrm{E}-03$ & $2,34 \mathrm{E}-04$ & $3,13 \mathrm{E}+00$ \\
7 & 20,420352 & $3,30 \mathrm{E}-02$ & $2,34 \mathrm{E}-04$ & $4,38 \mathrm{E}+00$ \\
8 & 23,561945 & $1,13 \mathrm{E}-01$ & $2,78 \mathrm{E}-03$ & $5,83 \mathrm{E}+00$ \\
9 & 26,703538 & $3,20 \mathrm{E}-01$ & $3,02 \mathrm{E}-02$ & $7,48 \mathrm{E}+00$ \\
10 & 29,845130 & $7,47 \mathrm{E}-01$ & $1,46 \mathrm{E}-01$ & $9,30 \mathrm{E}+00$ \\
11 & 32,986723 & $1,52 \mathrm{E}+00$ & $4,69 \mathrm{E}-01$ & $1,13 \mathrm{E}+01$ \\
12 & 36,128316 & $2,50 \mathrm{E}+00$ & $1,11 \mathrm{E}+00$ & $1,33 \mathrm{E}+01$ \\
13 & 39,269908 & $6,23 \mathrm{E}+00$ & $2,86 \mathrm{E}+00$ & $1,53 \mathrm{E}+01$ \\
14 & 42,411501 & $8,91 \mathrm{E}+00$ & $5,02 \mathrm{E}+00$ & $1,73 \mathrm{E}+01$ \\
15 & 45,553093 & $1,26 \mathrm{E}+01$ & $8,15 \mathrm{E}+00$ & $1,89 \mathrm{E}+01$ \\
16 & 48,694686 & $1,46 \mathrm{E}+01$ & $1,05 \mathrm{E}+01$ & $1,99 \mathrm{E}+01$ \\
17 & 51,836279 & $5,31 \mathrm{E}+01$ & $3,79 \mathrm{E}+01$ & $2,01 \mathrm{E}+01$ \\
18 & 54,977871 & $5,98 \mathrm{E}+01$ & $4,56 \mathrm{E}+01$ & $1,91 \mathrm{E}+01$ \\
19 & 58,119464 & $7,05 \mathrm{E}+01$ & $5,72 \mathrm{E}+01$ & $1,68 \mathrm{E}+01$ \\
\hline
\end{tabular}




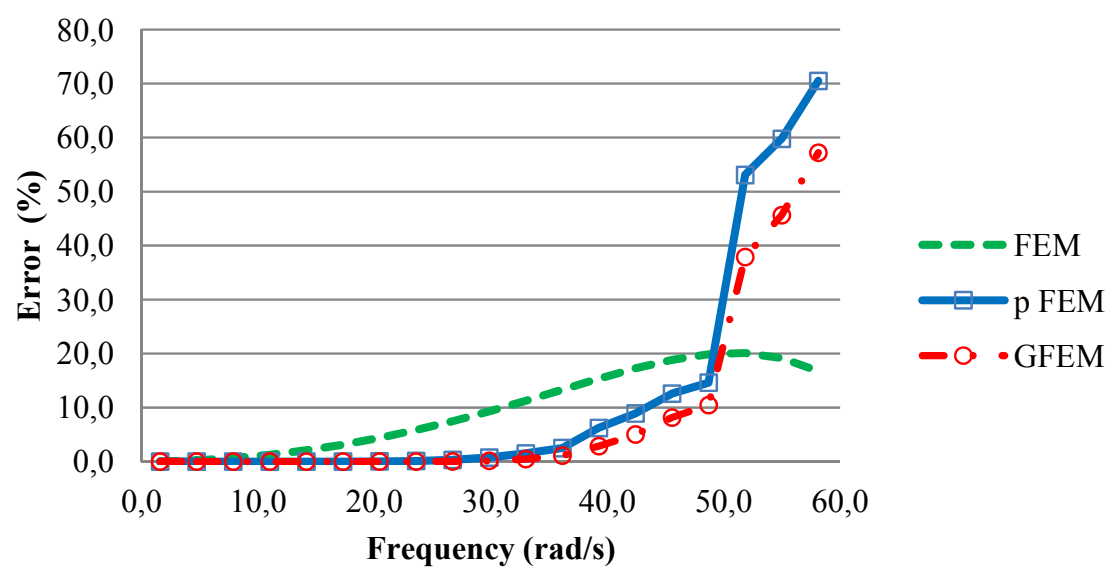

Figure 12. Relative errors (\%) obtained for the natural vibration frequencies.

From Table 2, we note that both the $p$ FEM and the GFEM obtained the same results when the first six modes were included in the analysis. However, the inclusion of the $7^{\text {th }}, 8^{\text {th }}$, $9^{\text {th }}$ and $10^{\text {th }}$ modes results in a better approximation by the GFEM.

This fact can be explained by observing the results presented in Table 3 . The $7^{\text {th }}, 8^{\text {th }}$, $9^{\text {th }}$ and $10^{\text {th }}$ modes were much better approximated with the GFEM than with the $p$ FEM. The $p$ FEM obtained better results until the $4^{\text {th }}$ mode, but the accuracy of the GFEM is good enough for these modes so that no difference is noted in the results from Table 2 in these cases.

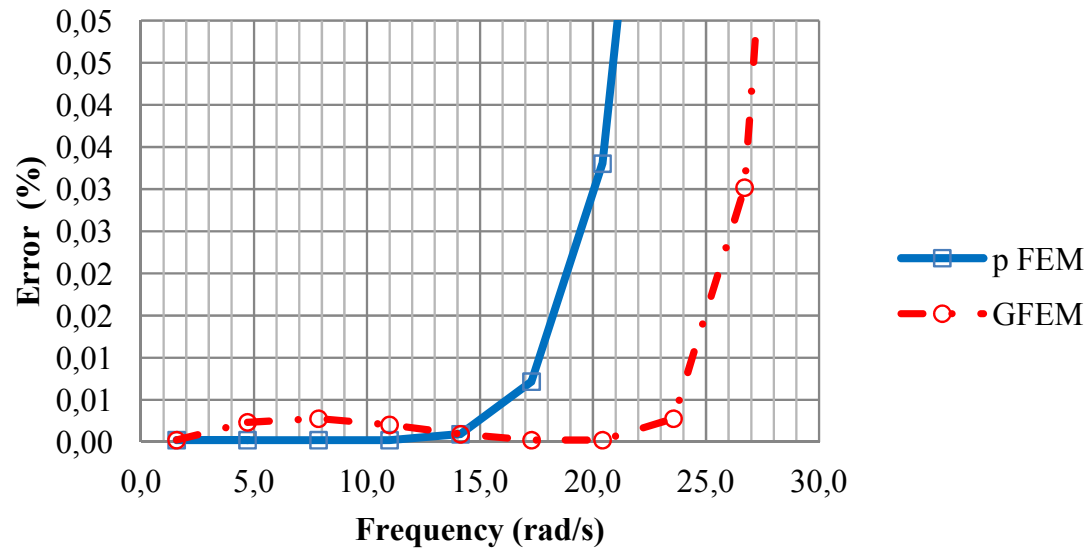

Figure 13. Relative errors (\%) obtained for the natural vibration frequencies.

These results indicate that the $p$ FEM is able to obtain better results for the first few vibration modes, but the GFEM is able to obtain better results for a larger frequency range. Since time response analysis makes use of several vibration modes at the same time, obtaining only a few vibration modes with high accuracy may not ensure an accurate time response. This is particularly true when the structure is subject to high frequency excitations. 
In this case, the GFEM is able to obtain better approximation for a larger range of vibration modes and then obtain better results for the time response analysis.

\section{CONCLUSION}

The main contribution of this work consists in formulating and investigating the performance of the Generalized Finite Element Method (GFEM) for vibration analysis of bars. The proposed generalized $\mathrm{C}^{0}$ element allows applying boundary conditions as in the standard finite element procedure. In some of the recently proposed methods such as the modified CEM [12], it is necessary to change the set of shape functions depending on the boundary conditions of the problem. In others, like the Partition of Unity used by [5] and [10], the boundary conditions are applied under a penalty approach. In addition the GFEM enrichment functions require less effort to obtain than the FEM shape functions in a conventional hierarchical $p$ refinement.

The GFEM results were compared with those obtained by the $h$ and $p$ versions of FEM and other enriched methods. The GFEM is quite accurate and its convergence rates are higher than those obtained by the $h$-versions of FEM and the enriched methods in free vibration analysis of bars. Although the $p$ refinement of GFEM has produced excellent results and convergence rates, the adaptive GFEM exhibits special skills to reach accurately a specific frequency.

In most of the free vibration analysis it is virtually impossible to get all the natural frequencies. However, in practical analysis it is sufficient to work with a set of frequencies in a range (or band), or with those which have more significant participation in the analysis. The adaptive GFEM allows finding a specific natural frequency with accuracy and computational efficiency. It may be used in repeated analyses in order to find all the frequency in the range of interest.

The adaptive GFEM shows fast convergence and remains stable after the third iteration with quite precise results for the target frequency. The results have shown that the adaptive GFEM is more accurate than the $h$ refinement of FEM and the CEM, both employing a larger number of degrees of freedom. The adaptive GFEM in free vibration analysis of bars has exhibited similar accuracy, in some cases even better, to those obtained by the $p$ refinement of FEM.

Thus the adaptive GFEM has shown to be efficient in the analysis of longitudinal vibration of bars, so that it can be applied, even for a coarse discretization scheme, in complex practical problems. Future research will extend this adaptive method to other structural elements like beams, plates and shells.

In time response analysis the most accurate results were obtained with the GFEM. The results obtained with the linear FEM are very poor in comparison to the GFEM and the $p$ version of the FEM. The results indicate that the $p$ FEM is able to obtain better results for the first few vibration modes, but the GFEM is able to obtain better results for a larger frequency range. Since time response analysis makes use of several vibration modes at the same time, obtaining only a few vibration modes with high accuracy may not ensure an accurate time response. This is particularly true when the structure is subject to high frequency excitations. 
In this case, the GFEM is able to obtain better approximation for a larger range of vibration modes and then obtain better results for the time response analysis.

\section{REFERENCES}

[1] Arndt M., Machado R. D., Scremin A., “An adaptive generalized finite element method applied to free vibration analysis of straight bars and trusses". J. of Sound and Vibration, $329,659-672,2010$.

[2] Babuska I., Banerjee U., Osborn J. E., "Generalized finite element methods: main ideas, results, and perspective". Technical Report 04-08, TICAM, University of Texas at Austin, 2004

[3] Bathe K. Finite element procedures. New Jersey: Prentice Hall, 1996.

[4] De S., Bathe K. J., "The method of finite spheres with improved numerical integration". Computers and Structures, 79, 2183-2196, 2001.

[5] De Bel E., Villon P., Bouillard Ph., "Forced vibrations in the medium frequency range solved by a partition of unity method with local information". Int. J. for Numerical Methods in Engineering, Vol. 62, 1105-1126, 2005

[6] Duarte C. A., Babuska I., Oden, J. T., "Generalized finite element methods for threedimensional structural mechanics problems". Computers and Structures, 77, 215-232, 2000.

[7] Duarte C. A., Oden J. T., “An h-p adaptive method using clouds”. Computer Methods in Applied Mechanics and Engineering, 139, 237-262, 1996.

[8] Engels R. C., "Finite element modeling of dynamic behavior of some basic structural members". J. of Vibration and Acoustics, 114, 3-9, 1992.

[9] Ganesan N., Engels R. C., "Hierarchical Bernoulli-Euller beam finite elements". Computers \& Structures, 43, 297-304, 1992.

[10] Hazard L., Bouillard P., "Structural dynamics of viscoelastic sandwich plates by the partition of unity finite element method". Computer Methods in Applied Mechanics and Engineering, 196, 4101-4116, 2007.

[11] Leung A. Y. T., Chan J. K. W., "Fourier $p$-element for the analysis of beams and plates”. J. of Sound and Vibration, 212, 179-185, 1998.

[12] Lu Z. R., Law S. S., "Discussions on composite element method for vibration analysis of structure". J. of Sound and Vibration, 305, 357-361, 2007. 
[13] Melenk J. M., Babuska I., "The partition of unity finite element method: basic theory and applications". Computer Methods in Applied Mechanics and Engineering, 139, 289314, 1996.

[14] Oden J. T., Duarte C. A. M., Zienkiewicz O. C., "A new cloud-based hp finite element method". Computer Methods in Applied Mechanics and Engineering, 153, 117-126, 1998.

[15] Pinchover Y., Rubinstein J., An introduction to partial differential equations. Cambridge: Cambridge University Press, 2005.

[16] Schweitzer M. A., "An adaptive hp-version of the multilevel particle-partition of unity method". Computer Methods in Applied Mechanics and Engineering, 198, 1260-1272, 2009.

[17] Strouboulis T., Babuska I., Copps K., "The design and analysis of the generalized finite element method". Computer Methods in Applied Mechanics and Engineering, 181, 43-69, 2000 .

[18] Strouboulis T., Copps K., Babuska I., "The generalized finite element method". Computer Methods in Applied Mechanics and Engineering, 190, 4081-4193, 2001.

[19] Strouboulis T., Zhang L., Wang D., Babuska I., "A posteriori error estimation for generalized finite element methods". Computer Methods in Applied Mechanics and Engineering, 195, 852-879, 2006.

[20] Sukumar N., Chopp D. L., Moes N., Belytschko T., "Modeling holes and inclusions by level sets in the extended finite-element method". Computer Methods in Applied Mechanics and Engineering, 190, 6183-6200, 2001.

[21] Sukumar N., Moes N., Moran B., Belytschko T., "Extended finite element method for three-dimensional crack modeling". Int. J. for Numerical Methods in Engineering, 48, 1549-1570, 2000.

[22] Torii A.J., Machado R.D. "Transient dynamic structural analysis of bars and trusses using the generalized finite element method". Mecánica Computacional. 24:1861-1877, 2010 .

[23] Zeng P., "Composite element method for vibration analysis of structures, part I: principle and C ${ }^{0}$ element (bar)". J. of Sound and Vibration, 218, 619-658, 1998.

[24] Zeng P., "Composite element method for vibration analysis of structures, part II: C" element (beam)". J. of Sound and Vibration, 218, 659-696, 1998. 